muB eine wässerige Salzlösung vorhanden sein, deren Salze die osmotische Reaktionsfähigkeit der Zelle ermöglichen. Nach F. Hofmeister ${ }^{10}$ ) wären auch die Fermente in das Innere des Tropfens eingeschlossen.

Zu einer ganz ähnlichen Vorstellung kam L. Rhu mbler 1902, 379: , Da nun dic Alveolenwände für Wasser durchlăssig, für viele gelöste Substanzen halbdurchlässig sind, und der Alveoleninhalt gelöste Substanzen enthält, so muB in jeder Zellalveole ein gewisser osmotischer' Druck zustande kommen. . Dieser osmotische Druck in jeder Einzelalveole wird innerhalb des ganzen Alveolengefüges, soweit dieses im Gleichgewicht ist, überall derselbe sein; denn, wird in einer Alveole der osmotische Druck durch Auftreten einer neuen löslichen Substanz gröBer, so wird sogleich ein Wasserausgleich mit den Nachbaralveolen stattfinden und dadurch der osmotische Druck wieder auf eine gemreinsame Höhe zurückgebracht ${ }^{4}$.

Ich will mich mit diesem Hinweis auf die Folgerungen begnügen, die sich aus den Ver-

10) F. Hofme is ter, Die chemische Organisation der Zelle, Naturw. Rundsch. 1901, 581 (zit. nach R. Hoeber, loc. cit. 731 ). suchen ergeben. Mit der Annahme einer semipermeablen Plásmahaut sind die Ergehnisse unvereinbar. Das durch die Abbildung angedeutete Schema der Protoplasmagrundstruktur soll ebenfalls nur als Anregung dienen, eine morphologische Vorstellung von den physikalischen Vorgängen deș Zelleniebens auszubauen. In späteren ruhigen Zeiten ist es mir vielleicht vergönnt, meine Vorstellungen vom physikalischen Bau der Protoplasma eingehend zu begründen.

\section{Zusammenfas sung.}

1. Es gelingt, saure Farbstoffe intrazellulằn zur Ausflockung zu bringen.

2. Diese Ausflockung ist identisch mit der Wirkung von Neutralsalzen auf Semikolloide und wird als Dispersionsverringerung charakterisiert.

3. Der Vorgang beweist die Permeabilität der Zelloberfläche für Neutralsalze.

4. Die osmotischen Vorgänge spielen sich im Zellinnern ab.

5. Die gewonnenen Vorstellungen stehen im Einklang mit der Theorie von der Schaumstruktur des Protoplasmas.

\title{
Zur Theorie der Koagulationsgeschwindigkeit.
}

Von H. Freund li ch (Berlin-Zehlendorf). (Bingegangen am 1. Sept. 1918.)

Unsere Kenntnis der Koagulation und Koagulationsgeschwindigkeit hydrophober Sole, wie der der Metalle, der Sulfide, des $\mathrm{Fe}(\mathrm{OH})_{3}$, des $\mathrm{Al}(\mathrm{OH})_{3}$ u. a. ist neuerdings durch Arbeiten von R. Zsigmondy') und M. v. Smoluchowski2) sehr erhehlich erweitert worden. Bis dahin konnte man sich von diesem Vorgang etwa folgendes Bild machen: Voraussetzung für das Zusammentreten der Kolloidteilchen solcher Sole ist ihre Entladung; sie braucht nicht völlig zu sein, es genight, wenn ein gewisser Mindestwert der Ladung erreicht wirds). Die Entladung wird durch den Zusatz eines Elektrolyten bewirkt, und $z$ war ist in erster Linie dasjenige

1) R. Z slg mo n dy, Nachr. d. Konigl. Gesellsch. d.Wissensch. Göttingen 1917, 1; Zeitschr.f.Elektrochem. 23, 148 (1917).

2) M. v. Smoluchowski, Zeitschr. physik. Chem. 92, 129 (1917); Koll--Zeitschr. 21, 98 (1917); Physik. Zeitschr. 17, 587 (1916). (1915)

7) F. Powis, Zeitschr. f. physik. Chem. 89, 179
Ion des Elektrolyten von EinfluB, dessen Ladung der der Kolloidteilchen entgegengesetzt ist. Es scheint stets eine Aufnahme dieses wirksamen Ions durch die Kolloidteilchen stattzufinden, die nach ihrer Umkehrbarkeit und Abhängigkeit von der Konzentration und des Natur des Elektrolyten der Adsorption ähnelt 4). Wie weit diese Aufnahme wirklich eine Oberflächenverdichtung ist, wie weit sie als "elektrische Adsorption" aufzufassen ist, wird erst die immer noch fehlende ausführliche Untersuchung der Aufnahme von Elektrolyten - - und zwar sōwohl der Anionen wie der Kationen - durch fein verteilte amorphe Stoffe lehren. Jedenfalls wird nach den bisherigen Erfahrungen der gleiche Grad der Entladung und damit die gleiche Koagulationsgeschwindigkeit erreicht, wenn ä qui v-ale n te Mengen des wirksamen lons aufgenommen

4) H. Fre und 1 ich, Zeitschr. $f$ physik. Chem. 73 385 (1910); H. Freund lieh u. H. Sc in ti $\mathrm{cht}$, Zeitsehr. f. physik. Chem. 85, 641 (1913); J. A. Ga n n, Kolloidchem. Beih. 8, 64 (1916). 
worden sind ${ }^{3}$. Damit diese Menge an der Grenzfläche der Kolloidteilchen vorhanden ist, sind von stark adsorbierbaren Ionen - wie denen der Schwermetalle und organischen Kationen und Anionen - und von mehrwertigen lonen kleinere Konzentrationen in der Lösung nötig als von schwach adsorbierbaren und einwertigen. Erstere koagulieren daher viel stärker. Der besondere Zusammenhang zwischen den Fällungswerten - Konzentrationen einer bestimmt gewählten gleichen Koagulationsgeschwindigkeit - verschiedenwertiger anorganischer $\mathrm{Ka}$ tionen und Anionen läbt sich dadurch erklären, dab diese vielfach in a qui molarer Konzentration gleich stark adsorbiert werden; damit äquivalente Mengen an die Grenzfläche der Teilchen gelangen, sind bei der Gestalt der Adsorptionsisotherme sehr verschiedene Konzentrationen nötig ${ }^{6}$ ). Wie man sieht, hängt die eine besondere Eigentümlichkeit der Koagulation, die starke Abhängigkeit von der Wertigkeit der lonen, gar nicht mit der eigentlichen Koagulationsgeschwindigkeit - abgekürzt Ko.G. zusammen, sondern mit der Aufnahme einer bestimmten Menge des wirksamen lons und der dadurch bedingten Entladung, also mit einem umkehrbaren, statischen Vorgang. So kommt es, daB man denselben auffallenden Wertigkeitseinflub bei Erscheinungen wiederfindet, bei denen man keinen zeitlich verlaufenden Vorgang miBt, so bei dem Einfluß der Elektrolyte auf die Elektroendosmose und die Strömungspotentiale ${ }^{7}$.

Was den eigentlichen zeitlichen Verlauf der Koagulation anbetrifft, so schien er meist autokatalytisch $z u$ sein, und die Geschwindigkeit nahm ganz auffallend stark wit wachsender Elektrolytkonzentration zu. Der Zusammenhang $z$ wischen der Konstante $k$ der Ko.G. und der Konzentration c des Elektrolyten in der Lösung - nach erfolgter Adsorption - lieB sich in erster Annäherung durch eine Gleichung

$$
k=x \cdot c^{p}
$$

wiedergeben. Hier sind $x$ und $p$ Konstanten, und $z$ war hatte $p$ Werte zwischen 2 und $8^{8}$ ).

5) W. R.Whit ney u. J.E. Ober, Zeitschr. t. physik. Chem. 39, 630 (1902); siehe auch unter 4 .

6) H. Freund $11 \mathrm{ch}$ u. Mitarbelter loc. cit. S. 163.

7) G. v. Elissafoff, Zeitschr. f. physik. Chem. 79, 385 (1912); H. R. Kru y t, Koll.-Zeltschr. 22, 81 (1918)

g) H. H. Paine, Kolloidchem, Belh. 4, 24 (1912); Miyazawa, Journ. chem. Soc. Tokyo 38, 1179,1210 (1912); N. Is hizaka, Zeitschr. f. physik. Chem. 83, 97 (1913); J. A. Gann, loc. cit. S. 163
Nun der Portschritt durch die erwähnten Arbeiten von $R . Z$ sig mondy und M. v. S moluchowski. Zsigmondy fand, daB mit wachsender Elèktrolytkonzentration die Ko.G. nicht dauernd weiter wächst, sondern sie erreicht einen bestimmten Grenzwert, der von der Natur und der Konzentration des Elektrolyten unabhängig ist. Und für diese Geschwindigkeit der "raschen Koagulation" wies v. Smoluchowski nach, dah sie sich durchaus molekularkinetisch deuten lieb: er nimmt, einem Gedanken R. Zsig mo ndy's folgend, an, daB die entladenen Teilchen sich anziehen und zusammentreten, sobald je zwei oder mehr in die Wirkungssphäre ihrer gegenseitigen Anziehungskraft gelangen; berechnet man die Zahl dieser unelastischen Zusammenstöbe, so erhält man den richtigen Wert für die Geschwindigkeit der , raschen Koagulation “. Nach den bisherigen Versuchen reicht die Anziehungssphäre wenig über den doppelten Teilchenradius hinaus.

Offenbar ist demnach Gleichung 1 im Kerne unrichtig, denn sie läßt kein Konzentrationsgebiet voraussehen, in dem die Ko.G. von der Elektrolytkonzentration unabhängig ist. $\mathrm{Ob}$ auch, wie M. v. S moluch ows.ki glaubt, der autokatalytische Verlauf der "nicht raschen Koagulation" im Grunde der Koagulation nicht eigentümlich ist, sondern von Nebenumständen herrührt, soll später noch erörtert werden und vorerst dahingestellt bleiben. Die rasche Koagulation geht unweit des sog. isoelektrischen Punktes vor sich, in dem die Ladung null ist, bzw. in einem Konzentrationsgebiet in dessen Nähe, in dem die Ladung kleiner ist als ein bestimmter Mindestwert. Die nicht rasche Koagulation vollzieht sich in einem Gebiete kleinerer Konzentrationen und damit gröberer Ladungen, die gleichfalls unterhalb eines anderen Mindestwertes ${ }^{9}$ ) bleiben müssen. $M$. v. S moluchow ski erklärt die Abnahme der Ko.G. mit sinkender Elektrolytkonzentration damit, daß „im Falle unvollständiger Entladung der Doppelschicht die Anziehungskräfte der Wirkungssphäre so weit abgeschwächt seien, dab von den unmittelbaren Zusammenstößen nur ein gewisser - von der Elektrolytkonzentration abhängiger Bruchteil eine sofortige Verbindung derselben bewirkt".

Ich glaube nun, dab man bei einer etwas abgeänderten Auschauungsweise das aulfallende Ansteigen der Ko.G. mit wachsender Elektrolytkonzentration gut verständlich machen kamn,

9) Wenn von "kritischem Mindestwert der Ladung" die Rede ist, so ist im allgemeinen dieser gemeint. 
die bei M. v. Smoluchowski keine Erklarung findet. Er nimmt an, dab die Anziehungskraft in der Wirkungssphäre durch die Ladung verringert wird. Man kann nun vielleicht as dessen Stelle annehmen, daß die Anziehungskraft selbst unverändert bleibt, das aber infolge der Abstobung $z$ wischen den Teilchen bei groberer Ladung die Zu. sammenstöbe nicht alle unelastisch sind, sondern nur ein Bruchteil der. selben, bei denen der Zusammenstob mit einer genügenden Wucht erfolgt is t. Nimmt man zunächst an, dab die Teilchen des Sols sich bloB nach ihrer Geschwindigkeit, nicht nach ihrer Masse unterscheiden, so wird für die Beziehung zwischen der Zahl der Teilchen und ihrer Geschwindigkeit die Maxwell: sche Verteilungsfunktion gelten. Je groBer nun die Abstobung $z$ wischen den Kolloidteilchen, um so gröBer die kritische Geschwindigkeit, die gerade genügt, dab der Zusammensto $B$ unelastisch wird und zur Koagulation führt. Entsprechend kleiner ist also auch die Zahl der Teilchen, die diese kritische, bzw. eine größere Geschwindigkeit besitzen. Die Ko.G. ist aber dieser Zahl proportional.

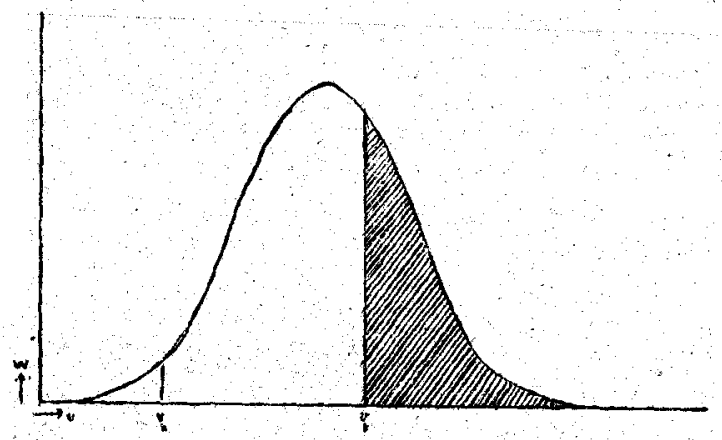

Fig. 1

Dies läbt sich gut im Bilde anschaulich machen. In Fig. 1 sind in bekannter Weise die Geschwindigkeiten $v$ der Kolloidteilchen als $\mathrm{Ab}$ szissen aufgetragen, ais Ordinaten die relativen Wahrscheinlichkeiten $W$, daß ein Teilchen eine Geschwindigkeit $z$ wischen $v$ und $v+d v$ besitzt; es ist also

$$
W=K v^{2} e^{-r}
$$

Pür die Zahl der Teilchen, die eine Geschwindigkeit in dem Bereich von $v$ bis $v+d v$ haben, gilt die Gleichung

$$
d n=x_{1} v^{2} e^{-\nabla^{2}} d v
$$

Die Fläche zwischen zwei Ordinaten der Fig. 1 ist also der Zahl der Teilchen proportional, deren Geschwindigkeit zwischen den zugehorigen Abszissen liegt.
Bei der Ladung null, im isoelektrischen Punkt, werden alle Teilchen, auch die langsamsten - der gesamten von der Kurve umschlossenen Fläche der Fig. 1 entsprechend unelastisch zusammenstoBen, da keine AbstoBung vorhanden ist. Auch bei kleinen Ladungen wird letztere noch so gering sein, dab wohl blob die Telichen als unwirksam wegfallen, deren Geschwindigkeit etwa kleiner ist als $v_{k}^{\prime}$. Ihrer geringen $Z a h l$ gemaß wird dieser Wegfall für die Ko.G. so gut wie ohne EinfluB sein. Dieser Umstand erklärt wohl, weshalb eine rasche Koagulation nicht bloB im isoelektrischen Punkt statt hat, sondern schon in einem Gebiet von Konzentrationen, in dem die Ladung unterhalb eines gewissen Mindestwertes bleibt. Ein starker Abfall der Ko.G. tritt erst ein, wenn die Ladung so groB geworden ist, dab die mittlere Geschwindigkeit nicht mehr genügt, um die Abstobung zu überwinden. Die kritische $\mathrm{Ge}$ schwindigkeit hat dann etwa den Wert $v_{k}$, und nur die Teilchen $\Sigma_{n}$ im schraffierten Teil kommen für die Ko.G. in Betracht. Man sieht, wie mit wachsender Ladung und wachsender kritischer Geschwindigkeit die Zahl der noch wirksamen Teilchen ungeheuer stark abnimmt und damit auch die Ko.G.

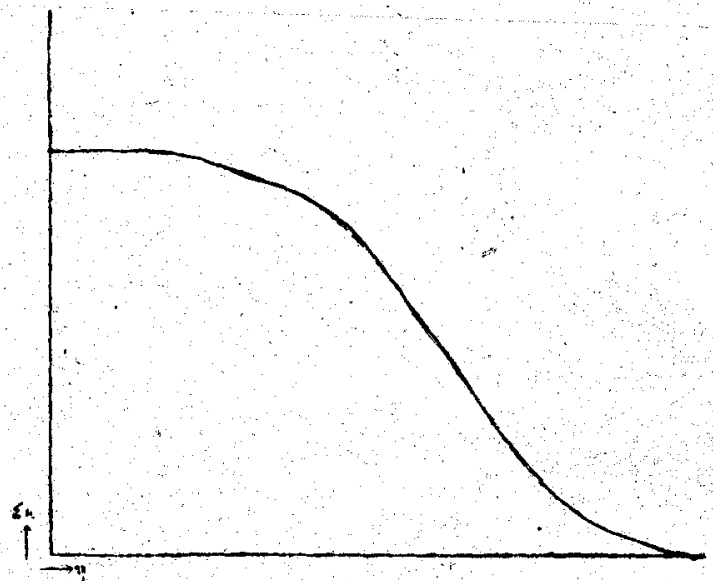

Fig. 2

Noch deutlicher wird dies, wenn man die Werte von $\sum_{n}$ den $v_{k}$-Werten zuordnet (siehe Fig. 2). Man erhält so eine .Spitzbogenkurve*, wie sie in der Vererbungslehre eine Rolle spielt. Die Konstanz des $\Sigma_{n}$ bei kleinem $v_{k}$, der starke Abfall bei gröBerem entspricht fetzt unmittelbar dem Verhalten der Ko.G.

Man hat also die nachfolgenden Beziehungen in Ansatz zu bringen:

1. Die Konstante der Ko.G. $k$ ist der Zahl der wirksamen Teilchen In proportional. 
2. In hängt nach der ,Spitzbogenkurve von der kritischen Geschwindígkeit $v_{k} a b$.

3. va wächst mit der Ladung der Teilchen \&.

4. $\varepsilon$ hăngt von der Elektrolytkonzentration in der Lösung $c$ ab.

Zu 4 ist zu bemerken, dab der Zusammenhang $z$ wischen der Ladung eines Kolloidteilchens und der Elektrolytkonzentration der Lösung und $z$ war handelt es sich natürlich stets um die im Adsorptionsgleichgewicht stehende Konzentration - recht verwickelt und wenig geklärt ist. F. Pow i ${ }^{10}$ ) hat vor allem die Ladung bzw. das Potential "I) suspendierter Oeltröpfchen mit Hilfe der Kataphorese in seiner Abhängigkeị von der Elektrolytkonzentration gemessen, und seine Ergebnisse werden durch die Untersuchung H. R. Kruyt's ${ }^{12}$ ) über den EinfluB der Elektro-

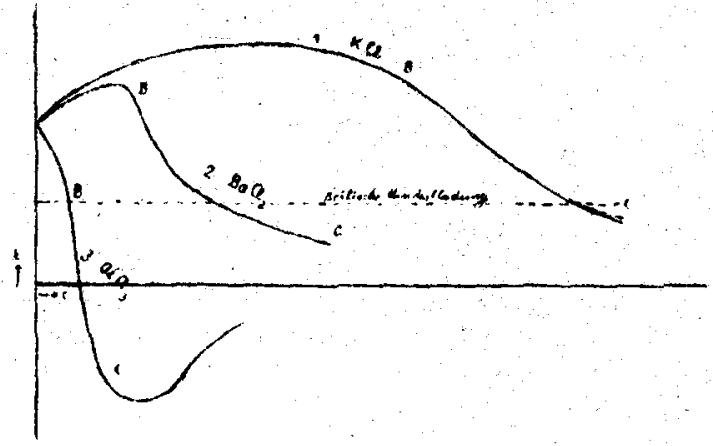

Fig. 3

lyte auf Strömungspotentiale in Glaskapillaren durchaus bestätigt. Kruyt zeigt weiter, daß auch die Messungen von G. v. El is s a f of $f^{13}$ ) über die Abhängigkeit der Elektroendosmose von der Elektrolytkonzentration mit diesen $\mathrm{Er}$ fahrungen in Einklang stehen. Nach diesen Untersuchungen läBt sich die Abhängigkeit des \& von der Elektrolytkonzentration c durch eine Kurve der Fig. 3 darstellen ${ }^{14}$ ). Die ersten kleinen Elektrolytmengen bedingen eine geringe Auf-

10) F. Pow is, loc.cit. $\$ .163$; auBerdem auch Zeitschr. f. physik. Chem. 89,91 u. 186 (1915).

ii) Die Ladung eines Kolloidteilchens und sein Potential E gegen die umgebende Lósung sind einander proportional. Betrachtet man die Doppelschicht eines kugelformigen Teilchens, so hat man

$$
E=\frac{\left(R_{2}-R_{1}\right)}{D \cdot R_{1} \cdot R_{a}}
$$

Hier sind $R_{t}$ und $R_{t}$ die Radien der inneren und auberen Belegung der Doppelschicht, $\mathrm{D}$ die Dielektrizitatskonstante.

12) H. R. Kru yt, loc. cit. S. 164.

18) G. v. Elissaf off, loc. cit S. 164.

14) Sie sind den Messungen H. R. K ruyt's entnommen und stellen die Abhängigkeit der Ladung von der Elektrolytkonzentration fïr $\mathrm{K} \mathrm{Cl}, \mathrm{BaCl}_{2}$ und $\mathrm{AlCl}$, bei Strömungspotentialen in Gilaskapillạren dar. ladung der Teilchen, erst dann folgt bei grö6eren Konzentrationen die Entladung, dic entweder bei den schwach adscrbierbaren und koagulierenden lonen allmäblich dem Nullwert des Potentials näher führt, oder aber bei den stark adsorbierbaren und koagulierenden lonen nach dem Durchschreiten des Nullwertes eine Aufladung im entgegengesetzten Sinne bewirkt; vielfach kommt weiter ein Minimum zu Stande, und die Ladung strebt dann von neuem dem Nullwert zu (siehe Kurve 3 in Fig. 3). Wie dieser verwickelte Verlauf zu erklären ist, steht noch dahin; wahrscheinlich handelt es sich um eine Wechselwirkung zwischen der eigentlichen Adsorption, der Oberflächenverdiclitung also, und elektrischen Einflüssen.

Für die hier vorliegenden Fragen ist nur das Stück BC dieser Kurven von Belang, das Gebiet der Entladung, das sich bis in die Nähe des isoelektrischen Punktes erstreckt. Es läBt sich genügend durch eine Gleichung

$$
\varepsilon=x_{2} \ln \frac{\gamma}{c}
$$

ausdrücken. $y$ ist eine Konstante, die man von Elektrolyt zu Elektrolyt verschleden wăhlen muB; es ist ja die Konzentration, bei der die Ladung der Teilchen null wird. Bei den bisher untersuchten Fällen von Ko.G. hat man sich in einem Konzentrationsgebiet vor dem isoelektrischen Punkt bewegt; demgemäB ist stets $\gamma>c$. Mir scheint es wahrscheinlich, daB man $\%_{2}$ für Elektrolyte mit lonen gleicher Wertigkeit nicht zu verschieden nehmen darf, wenn auch, soweit ich sche, nicht ausgeschlossen ist, dab in $x_{2}$ charakleristische Faktoren eingehen, wie in die Gleichung für die Diffusionspotentiale die Beweglichkeiten der Jonen. Eine logarithmische Abhängigkeit der Ladung von der Elektrolytkonzentration einzuführen, ist ja nur berechtigt, nach allem, wás man vơn der Abhängigkeit des Potentials - und der in unserem Fall diesem proportionalen Ladung - von der Konzentration weiB. Dab dieser Ansatz blob vorläufig gemacht wird, um weiter rechnẹn zu können, brauchi kaum betont zis werden.

Die kritische Geschwindigkeit $v_{k}$ wird man der Ladung zunächst proportional setzen. Betrachtet man die Kolloidteilcben als geladene Punkte, so gilt ja als Bedingung für $v_{k}$

$$
\frac{m v_{k}^{2}}{2} \geq \frac{\varepsilon^{2}}{r}
$$

hier ist $\mathrm{m}$ die Masse der Teilchen, $\mathbf{r}$ der miltlere Abstand, der für die Abstoßung in Frage kommt. Auch dies ist natürlich nur eine angenäherie Betrachtungsweise, und man mub 
mit der Moglichkeit rechnen, daß die Ladungen auf der Oberfläche der Kolloidteilchen unter Uniständen nicht gleichmäfig verteilt sind, und daB es z. B. etwas ausmacht, ob beim $\mathrm{Zu}$ sammensto $\mathrm{B}$ zweier Teilchen die Ladungen sich genau gegenüber liegen oder nicht. Die Gestalt der Teilchen kommt gleichfalls in Betracht; bei den später berücksichtigten Solen wird man aber wohl mit kugelförmigen Teilchen rechnen dürfen. Wir setzen also angenähert

$$
v_{k} \geqq x_{3} \varepsilon
$$

wo $x_{3}$ ein Proportionalitätsfaktor ist.

Die Geschwindigkeit der Teilchen $-v$ ist wit der Zahl der Teilchen dn, deren Geschwindigkeit $z$ wischen $v$ und $v+d v$ liegt, durch die oben erwăhnite Gleichung

$$
\mathrm{dn}=x_{1} \mathrm{v}^{2} \mathrm{e}^{-v^{2}} \mathrm{dv}
$$

verknüpft. Um nưn sämtliche Teilchen zu erhalten, deren Geschwindigkeit gleich oder gröber ist als $v_{k}$, muB man die rechte Seite von 4 von $v_{k}$ bis $\infty$ integrieren. Man erhält

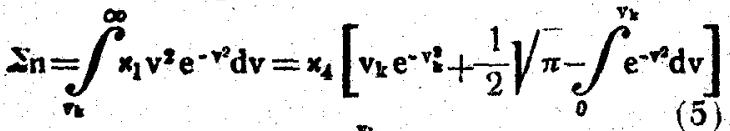

Das Integral $\quad 1=\int_{0}^{p_{x}} e^{-r^{2}} d v$

läBr sich nicht weiter auswerten; man findet Zahlenwerte dafür in verschiedenen Tabellenwerken ${ }^{15}$ ).

Die Konstante der Ko.G. k wird man der In proportional setzen

$$
k=x_{5} \Sigma_{n}
$$

Man kann ja gewissermaßen annehmen, daB im Gebiet der nicht raschen Koagulation die Zabl der in jedem Augenblick koagulierbaren Teilchen, also ihre Konzentration, geringes ist als die Gesamtzahl. Die Ko.G.-Konstante, als reziproker Wert der Koagulationszeit für gleiehen Umsatz, ist" der Konzentration, also der $\Sigma$, proportional, da ja nach M. v. S moluchowskj die Ko.G. weitgehend das Gepräge einer Reaktion zweiter Ordnung hat und bei dieser die genannte Proportionalität zur Konzentration vorhanden ist.

Setzt man die Werte von 2,3 a und 5 in 6 ein und vereinigt die zusammentretenden Konstanten zul zwei neuen Konstanten $\lambda_{1}$ und $\lambda_{2}$, so ergibt sich

$k=\lambda_{2}\left[\lambda_{1} \ln \frac{\gamma}{c} e^{-\left(\lambda_{1} \ln \frac{\gamma}{v}\right)^{v}}+\frac{1}{2} \sqrt{\pi}-\int_{0}^{\lambda_{1} \ln \frac{\gamma}{\mathrm{c}}} \mathrm{e}^{-\gamma^{2}} \mathrm{dv}\right]$

15) Benutzt wurden E. Jahnke und F. Emde, Funktionentafein (B. G. Teubner, Leipzig und Berlin 1909).
Bequemer ist es mit der Gleichuing

$$
k=\lambda_{2}\left[v_{k} e^{-v_{k}^{2}}+\frac{1}{2} \sqrt{\pi}-\int_{0}^{v_{k}} d v\right]
$$

zu rechnen. Es ist

$$
\lambda_{1}=x_{2}, x_{3} \text { und } \lambda_{2}=x_{4}, x_{5}
$$

Da $x_{3}$ ebenso wie $x_{2}$ nicht von der Natur des Elektrolyten abhängt, so gilt dies auch von $\lambda_{1}$.

Was nun die Prüfung der Formel anbetrifft, so sieht man zunächst, daß für den Grenzfall $v_{k}$ gleich null, also für Werte von c gleich $\gamma$, das erste- und dritte Glied der Klammer null werden, folglich

$$
k_{0}=\frac{1}{2} \lambda_{2} \sqrt{\pi}=\text { konst.: }
$$

Wir haben die konstante Geschwindigkeit der ras chen Koagulation.

Für unendlich grobe Werte von $v_{k}$ und unendlich kleine von $c$ wird das erste Glied der Klammer gleichfalls null, für das dritte gilt

$$
\int_{0}^{\infty} e^{-v^{2}} d v=\frac{1}{2} \sqrt{\pi}
$$

es wird also die Klammer und damit auch $k$ gleich null. Bei kleinen Elektrolytkonzentrationen sind die Sole unbegrenzt beständig.

Dazwischen liegt das Gebiet der nicht rasch en Koagulation mit dem starken Anstieg der Ko.G. mit wachsender Elektrolytkonzentration. Die in diesem Gebiet ausgeführten Messungen lassen viel zu wünschen übrig. Bei dem von H. H. Pa in e ${ }^{16}$ ) benutzten Kupfersol (richtiger Kupferoxydsol) hat man es mit einem ziemlich empfindlichen, unbeständigen Sol zu tun, und das Mefverfahren ist an sich nicht gerade genau; allerdings ist es tatsächlich die Ko.G., die er mift, wenn man von der Störung ahsieht, die das Rühren bewirkt. Das von Miyazawa ${ }^{16}$, N. Ishizaka' verwandte $\mathrm{Al}(\mathrm{OH})_{3}$-Sol ist sehr viel beständiger, und die von ihnen verfolgte Zähigkeítszunahme bei der Koagulation ist weit zuverlässiger zu bestimmen. Aber es steht nicht fest, wie weit man wirklich mit der Geschwindigkeit der Zähigkeitszunahme die Ko.G. miBt. Bei stark koagulierenden Anionen ist der Parallelismus zwischen beiden Erscheinungen sicher recht unvollkommen, und man kann nur für die schwach koagulierenden anorganischen Anionen annehmen, daB der Zähigkeitszunahme ein proportionaler Betrag koagulierter Teilchen entspricht. Dafür läbt sich anführen, daB die Ko.G.-Kuryen für verschiedene Konzentrationen eines Elektrolyten

10) H. H. Paine, Miyazawa, N Ishizaka u. J. A. Gann, loc. cit. S. 164 . 
bei gleichem $\mathrm{Al}(\mathrm{OH})_{3}$. Gehalt des Sols einander affin sind, wie es die Theorie M. v.Smoluchowski's fordert; d. h. die Abszissen, also Zeiten, der Kurve für die Elektrolytkonzentration 1 lassen sich durch Multiplikation mit einer bestimmten Zahl in die Abszissen der Kurve für die Elektrolytkonzentration 2 umwandeln. Der reziproke Wert dieser Zahl, der Fak tor der Ko.G. V kann ohne weiteres als MaB für die Ko.G. genommen werden, wobei man ihn für die Kurve mit der kleinsten Elektrolyrkonzentration gleich 1 setzt. Man kann mit Hilfe von $V$ die Abhangigkeit der Ko.G. von der Elektrolyts, konzentration angeben auch dann, wenn man den gesamten zeitlichen Verlauf der Koagulation nicht durch eine Gleichung hat ausdrücken können. Hat man dagegen eine Geschwindig. keitsgleichung aufgestellt, so kann man die Geschwindigkeitskonstante $\mathrm{k}$ als $\mathrm{MaB}$ für die Ko.G. verwenden. $k$ ist, als reziproke Zeit für einen bestimmten Umsatz, dem V proportional, vorausgesetzt, daB die Zeit in der ersten Potellz in die Geschwindigkeitsgleichung eingeht, wás für die in den nachfolgenden tabellen berücksichtigten Fälle zutrifft. $k$ und $V$ lassen sich also ohne Unterschied verwenden '7).

In den nachfolgenden Tabellen finden sich demgemăB sowohl Werte von $k$ wie von $V$. Es wurden wesentlich Messungen mit schwach koagulierenden anorganischen Elektrolyten gewählt.

Tabelle l.

$\mathrm{KCl} ;{ }^{\circ} \mathrm{Al}(\mathrm{OH})_{\mathbf{3}}-\mathrm{Sol}$ (J. A. Gann ${ }^{18}$ ) $\gamma=200 ; \lambda_{1}=1,737 ; \lambda_{2}=0,07714$.

\begin{tabular}{c|c|c}
\hline c (Millimol i. L.) & $k$ (beobachtet) & $k$ (berechnet) \\
\hline 60 & 0,0023 & 0,0022 \\
70 & 0,0049 & 0,0057 \\
80 & 0,011 & 0,011 \\
100 & 0,031 & 0,028
\end{tabular}

Tabelle J.

K-Benzoat; Al(OH) $)_{3}$ Sol (J. A. Gann' ${ }^{19}$ ). $\gamma=52,97 ; \lambda_{1}=1,737 ; \lambda_{2}=1,333$.

\begin{tabular}{c|c|c}
\hline c (Millimol i. L.) & $k$ (beobachtet) & $k$ (berechnet) \\
\hline 9,634 & 0,00075 & 0,00062 \\
11,63 & 0,0036 & 0,0037 \\
12,63 & 0,0067 & 0,0070 \\
13,62 & 0,012 & 0,013 \\
14,62 & 0,024 & 0,022 \\
& &
\end{tabular}

17) Siehe H.H.Paine u. N.I shiza ka, loc. cit. 164.

18) J. A. Gann, Tabelle 53, S. 122 der auf S. 263 erwahnten Ablandlung.

10) J. A. Ga nn, Tabelle 54, 5. 122.
Tabelle 111 .

$\mathrm{NH}_{4} \mathrm{Cl} ; \mathrm{Al}(\mathrm{OH})_{3}-\mathrm{Sol}$ (N. Is hizaka ${ }^{20}$ ).

$\gamma=100 ; \lambda_{1}=1.737 ; \lambda_{2}=17,83$.

\begin{tabular}{c|c|c}
\hline c. (Millimol i. L.) & V (beobachtet) & V(berechnet) \\
\hline 38,0 & 1 & 0,94 \\
41,7 & 2,5 & 3,2 \\
45,5 & 5 & 4,6 \\
55,6 & 9 & 8,8
\end{tabular}

Tabelle IV.

$\mathrm{NaCl}$; Kupfersol (H. H. Paine ${ }^{21}$ ). $y=7,586 ; \lambda_{1}=1,737 ; \lambda_{2}=71,84$.

\begin{tabular}{c|c|c|}
\hline c (Millimol i. 1.) & V (beobachtet) & V(berechnet) \\
\hline 2,012 & 1 & 0,9 \\
2,263 & 2,28 & 2,0 \\
2,515 & 3,5 & 3,9 \\
2,767 & 6,2 & 6,7 \\
3,269 & 11,9 & 15 \\
3,772 & 26 & 26 \\
4,275 & 41,1 & 37
\end{tabular}

Die Reihe mit Kaliumbenzoat wurde hinzugefigh, um doch noch einen etwas andersartigen Elektrolyten mit stark adsorbierbarem und koagulierenden Anion zu berücksichtigen; das affine Verhalten der Kurven ist bei diesem Salz ausreichend erfüllt. Die Konstanten $\gamma$ und $\lambda_{1}$ wurden durch Probieren gefunden.

Es läbt sich also wohl sagen, daB die Art des Anstieges der Ko.G. mit der Elektrolytkonzentration durch wahrscheinliche Werte der Konstanten wiedergegeben werden kann; es ist zu bedenken, $\mathrm{da} B$ die Werte von $k$ und $V$ meist blos auf $10-20$ Proz. sicher bestimmt sind.

Mit wachsendem $c$ nähert sich das $k$ noch in einigem Abstand von $y$ stark dem $\mathrm{k}_{0}$. Für den Fall der Tabelle $1 \mathrm{z}$. B. wird für $c=186,2$ Millimol $k=0,06827$, für $c=190,5 k=0,06834$; da $k_{0}=0,06837$, so unterscheiden sie sich von ihm nur um $1 \frac{1 / 2}{}$ bzw. $1 / 2$ pro Tausend. Für $c=178$ ist $k$ bereits auf 0,06349 , also um 7 Proz., für $c=170$ auf 0,05718 , also um 16,5 Proz., gesunken.

Mit sinkendem c nähert man sich rasch sehr kleinen Ko.G. Wählt man wieder den Fall der Tabelle 1 als Beispiel, so wird für $\mathrm{c}=\mathbf{3 0}$ Millimol $\mathrm{k}=5 \cdot 10^{-6}$, für $\mathrm{c}=20$ Millimol $\mathrm{k}=3 \cdot 10^{-8}$. Nun wurde für 60 Millimol $-\mathbf{k}=0,0023$ der halbe Umsatz - d. h. die Hälfte der gesamten Zähigkeitszunahme - in 2 Stunden durchmessen; demgemäb für $c=30$ in 40 Tagen,

20) N. Is sizak a, Tabelle 29, S. 117 der aul S. 164 erwăhnten Abhandlung.

21) H. H. Paine, Tabelle 2, IV , S. 43 der auf S. 164 erwalinten Abhandlung. 
fur $c=20$ in 15 Jahren. J. A. $G$ a n n konnte bei 10 Millimol $\mathrm{KCl}$ in mehreren Tagen keine Veranderung des $\mathrm{Al}(\mathrm{OH})_{3}$-Sols beobachten. In der Grobenordnung gut stimmt auch folgende Erfahrung ${ }^{22}$ ): $A s_{2} S_{3}$-Sol hat für $\mathrm{KCl}$ einen -Fällungswert" von etwa 70 Millimol, d, h. in etwa 2 Stunden - eigentlich wohl nach der Art derVersuchsausführung in einer viel kürzeren Zeit - wird es in einer Lösung von $70 \mathrm{Milli-}$ mol $\mathrm{KCl}$ völlig koaguliert. Bei einer $\mathrm{KCl}$-Konzentration von etwa 4 Millimol wird in Laufe von 11 Monaten eine fast völlige Koagulation erreicht, bei einer von 1 Míiìmoi ist im Laufe von 11 Monaten so gut wie keine Koagulation zu bemerken. Der ungeheure Anstieg der Konzentrationsabhängigkeit bedingt eben das Auftreten eines "Schwellenwertes".

Uebrigens ersieht man aus der Gleichung 8, daB man die Konstante der raschen Koagulation $k_{0}$ aus Messungen der nicht raschen Koagulation berechnen kann; aus letzteren ergibt sich ja nach Gleichung 7 die Konstante $\lambda_{2}$.

Die bisher der Einfachheit wegen gemachte Einschrärikung, daB die Kolloidteilchen gleiche Masse haben sollten, ist nicht notwendig. Hat man ein polydisperses Sol, d. h. eins mit Teilchen yon verschiedener Masse, so werden sich die Teiichen nun bezüglich ihrer Bewegungsenergie nach der $\mathrm{M}$ a $\times$ well'schen Funktion verteilen lassen, und die Zahl der Teilchen, die eine bestimmte kritische Bewegungsenergie haben, oder eine noch großere, werden für die Ko.G. im Gebiet der nicht raschen Koagulation maBgebend sein. Und diese kritische Bewegungsenergie laBt sich zur Ladung und zur Masse in Beziehung setzen gemäB der Gleichung 3.

Soweit ich sehe, führen andere Anschauungen gleichfalls zu einem ahnlichen Zusammenhang zwischen Ko.G. und Elektrolythonzentration, z. B. die von R. Zsig m ondy ${ }^{23}$ ) angedeutete. Wenn ich recht verstehe, nimmt er zur Erklärung der nicht raschen Koagulation wenigstens in der Nahe des Schwellenwertes an, dab die Ladung der Teilchen nicht streng konstant bleibt, sondern Schwankungen unterliegt, so daB auch Teilchen vorkommen, deren Ladungen unterhalb des kritischen Mindestwertes liegen und daher zu unelastischen Zusammenstößen führen. Die Zahl solcher Teilchen wird um so kleiner sein, je gröfer der Abstand der

27 H. Freandich, Zeitschr. f. physik. Chem. 44, 144 (1903).

23) R. Zsigmondy, Nachr. d. Konigl. Geselisch. d. Wissensch. Göttingen 1917, 16. mittleren Ladung von der kritischen. Die Fehlerkurve wird wohl für die Abhängigkeit der Häufigkeit solcher Teilchen von diesem Ladungsabstand und damit von der mittleren Ladung gelten, und die Ladung hängt wie bisher von der Konzentration ab. -

Wie oben erwähnt, neigt v. Smoluch ow ski der Meinung $z u$, daB der vielfach beschriebene autokatalytische Verlauf der nicht raschen Koagulation durch Nebenumstănde verursacht sei. Er zieht zwei in Betracht. Einmal die Tatsache, daß Rähren das Zusammenballen der Teilchen stark begünstigt, sobald sie eine gewisse Gröbe erreicht haben, während kleinere Teilchen wenig oder gar nicht davon beeinfluBt werden. Diesen Umstand berücksichtigt er bei der von $\mathrm{Paine} \mathrm{e}^{24}$ ) untersuchten Koagulation des Kupfersols. Ihm ist wohl z. T. auch zuzuschreiben, daB für den Augenschein die Koagulation vieler Sole so durchaus autokatalytisch verläuft. Wenn man 2. B. ein $\mathrm{As}_{2} \mathrm{~S}_{\mathrm{g}}$-Sol mit einem stark koagulierenden Elektrolyten (etwa einem Salz mit dreiwertigem Kation) in so kleiner Konzentration versetzt, daB der Schwellenwert nicht allzuviel überschritten wird, und rührt um, so bleibt das Sol eine Zeit lang anscheinend völlig klar, um sich auf einmal plötzlich milchig zu trüben und die Plocken fallen zu lassen. Offenbar bedingt das Rühren hierbei eine schnelle Koagulation der Flocken, sobald sie eine gewisse Größle exreicht haben.

Etwas anders liegt es beim $\mathrm{Al}\left(\mathrm{OH}_{33}-\mathrm{Sol}{ }^{25}\right)$. Dies ist weit hydrophiler als das eben genannte Kupfersol und wird bei der Koagulation sehr $\mathbf{z a h}$, schlieblich oft gelartig. Hier ist der autokatalytische Verlauf vielleicht der Zähigkeitszunahme zuzuschreiben, die der Koagulations: zunahme nicht notwendig parallel geht. Das Autokatalytische kann $z$. B. dadurch $z u$ Stande kommen, dab die koagulierten Teilchen zunehmend mehr Wasser in einen starren Verband aufnehmen, dadurch die Zahigkeit steigern, und da6 diese Wirkung erst deutlich zu Tage tritt, wenn die Kongulation einen bestimmten Betrag erreicht hat.

Nun spricht aber doch viel dafür, daB der autokatalytische Verlauf der nicht raschen Koagulation eigentümlich ist. Man beobachtet nämlich häufig, daB, auch wenn nicht geriihrt wird, eine kolloide Lösung bei der Koagulation erst längere Zeit klar bleibt und sich dann sehr plötz-

24) H. H. Pa ine, loc. cit. S. 164.

25) Versuche von N. Is hizaka u.J. A. Gann loc. cit. S. 164 . 
lich triibt und ausflockt ${ }^{26}$ ). Dann sei an die von Goodwin ${ }^{27}$ ) und $C$. L. Wagner ${ }^{28}$ ) verfolgte langsame Hydrolyse des Eisenchlorids in wässeriger Lösung erinnert. Diese wird damit eriklärt, daf die Hydrolyse selbst rasch erfolgt, daß aber die erst abgeschiedenen, sehr kleinen kolloiden $\mathrm{Fe}(\mathrm{OH})_{\mathrm{B}}$-Teilchen sich langsam in gröbere umwandeln, die aber immer noch ultramikroskopisch sind. Der Verlauf des Vorganges wurde mit Hilfe der Leitfăhigkeit gemessen, indem die zunächst sehr reichlich von den Teilchen adsorbierte $\mathrm{HCl}$ beim Gröberwerden an die lösung abgegeben wird. Dieser Verlauf ist nun ausgesprochen autokatalytisch. Da die Teilchen weit unterhalb der Gröbe bleiben, da 6 das Rühren eine Rolle spielen könnte, da ferner nicht einzusehen ist, wie in diesem Falle durch das Meßverfahren das Autokatalytische vorgetäuscht werden kann, möchte man es hier durchaus der Koagulation selbst zuschreiben.

Dies ist nun auch wahrscheinlich, falls die hier vertretene Erklärung des Elcktrolyteinflusses bei der langsamen Koagulation richtig ist. Die Möglichkeit, daß Teilchen, die bereits aus zwei oder dreien bestehen, noch nicht erheblich an Geschwindigkeit verloren haben, also bei ihrer gröBeren Masse eine größère lebendige Kraft haben und daher zu unelastischen Zusammen. stöBen Veranlassung geben, scheint recht groß zu sein. Die Beobachtung von (i. Wiegn e ${ }^{29}$ ) u. a., daß̣ sich tatsächlich kleinere Teilchen bei der Koagulation lieber an gröBere anlagern, fällt bestätigend ins Gewicht. Bei der raschen Koagulation ist von dieser Autokatalyse nichts $2 u$ bemerken, weil eben alle ZusammenstöBe unelastisch sind.

Uebrigens läbi auch M. v. Smolu ch owsk $i^{30}$ j die Möglichkeit offen, daB bei der nicht raschen Koagulation ein autokatalytischer Verlauf statthat. -

Die hier entwickelte Anschauung kann man auch auf eine andere Erscheinung übertragen, die bei offenbar grober Aehnlichkeit mit der Koagulation zunächst schwer mit ihr in Einklang zu bringen war. Es ist das der sog. Adsorptionsräckgang ${ }^{31}$ ) und sein eigen-

3) Z. B. die Versuche von A. Lottermoser an Wolframsäuresolen, Koll.-Zeitschr. 15, 145 (1914).

27) Goodwi n, Zeitschr. f. physik. Chem. 21, 1 (1896); Physik. Rev. 11, 193 (1900).

28) C. L. Wagner, Wien. Monatsh. 34, 95 (1913).

20) G. Wiegner, Koll.-Zeitschr. 8, 227 (1911).

*) M. v. S moluch ow ski, Zeitschr. f. physik. Chem. 92, 153 (1917).

si) H. Freundlich u. H. Schucht, Zeitschr. physik. Cliem. 85, 660 (1913): H. Freundlich u. E. Hase, Zeitschr. f. physik. Chem. 89, 415 (1915). tümlicher zeitlicher Verlauf. Die koagulierten Teilchen eines Sols, die sich völlig zu Boden gesetzt haben, befinden sich bei vielen Solen, z. B. denen der Sulfide und Hydroxyde, noch nicht in einem Gleichgewichtszustand. Es sind zunächst ganz lockere Flockell, in denen die einzelnen Kolloidteilchen oft so wenig fest verbunden sind, dab man sie durch bloBes Auswaschen wieder zu einem Sole peptisieren kann. UeberläBt man die Plocken in der Flüssigkeit sich selbst, so werden sie im Laufe der Zeit viel gröber, dichter; ja nehmen schließlich häufig ein kristallinisches Gepräge an. Die Abnahme an Oberfläche und Adsorptionsvermögen ist dabei unter Umständen so stark, daß der koagulierende Elektrolyt, der vou den Teilchen adsorbiert worden war, im Laufe dieses Vergröberungsvorganges wieder an die lösung zurückgegeben wird; es tritt ein Adsorptionsrückgang ein. Dieser Vorgang verläuft gleichfalls autokatalytisch und seine. Geschwindigkeit steigt - ebenso wie die Ko.G. - ganz auberordentlich stark mit der Konzentration des koagulierenden Elektrolyten an, so daß man auch hier die Gleichung 1 hat anwenden können.

Diese Erscheinung läßt sich jetzt sehr wohl folgendermaBen deuten: es handelt sich in der Tat um nichts anderes, als um die Fortsetzung der Koagulation. Damit die ursprünglichen Teilchen aneinander haften, genügt es, dab die Ladung derselben unterhalb eines Mindestwertes bleibt, eine völlige Entladung ist nicht erforderlich. Ebenso ist es nicht nötig, wie R. Zsig. mondy ${ }^{32}$ ) sehr mit Recht hervorhebt, daB die Tellchen sich unmittelbar berühren. Die Anzlehungskraft könnte sehr wohl soweit reichen, daB äußerst dünne Flüssigkeitshäutchen noch bestehen bleiben, weniger bei den Plocken von Metallsolen, wohl aber bei solchen der Sulfide. Hydroxyde u. a. m. Es entstehen unter diesen Umständen schon hinreichend große und schwere Flocken, daB sie sich unter dem EinfluB der Schwere absetzen Aber die noch vorhandenen Ladungen und Flüssigkeitshäute sind nicht ohne Bedeutung für das weitere Vergröbern der Teilchen, für das Verschmelzen der Einzelteilchen zu größeren, nicht mehr peptisierbaren Flocken In lockeren Verband der frisch gebildeten Flocken werden die Einzelteilchen noch immer schwin: gende Molekularbewegungen ausführen Auch jetzt wird die noch vorhandene Ladung eine Abstobung bedingen, die um so gröBer ist je gröBer die Ladung. Und nur die Teilchen, die

se) R. Z s igm on dy, Nache. d. Koruigl. Gesellsch. a. Wisseusch. Goltingen 1917, 33. 
hei ihren Schwingungen mit einer gewissen kritischen Wucht zusammenstoben, werden sich unmittelbar berühren, inniger vereinigen und verschmelzen. Die Geschwindigkeit der Vergrobberung und damit des Adsorptionsrückganges wird der Zahl dieser genügend bewegten Teilchen proportional sein, und man hat wiederum dic starke Zunahme der Geschwindigkeit mit der wachsenden $\mathrm{Zahl}$ dieser Teikchen bei abnehmender Ladung.

Man hat also hier beim Adsorptionsriickgang die gleiche Gedankenkette wie bei der Koagulation: die Geschwindigkeit des Adsorptionsrückganges ist proportional der Zahl der wirksamen Einzelteilchen in dem Verband der abgesetzten Flocken; diese Zahl hängt gemäB der Fehlerkurve von der kritischen Bewegungsenergie der Teilchen ab; die kritische Bewegungsenergie muß um so gröBer sein, fe gröher die Ladung der Teilchen ist; die Ladung hängt, wie bei der Koagulation, von der Konzentration des koagulierenden Elektrolyten ab.

Gut im Einklang mit dieser Auffassung ist noch folgende Tatsache: nur die Flocken eines Sols, die mit schwach koagulierenden lonen ausgeflockt worden sind, lassen - sich glatt und leicht durch Auswaschen wieder peptisieren, nicht solche, die mit stark koagulierenden lonen ausgefallt worden sind ${ }^{33}$ ). Nun ist nach den Messungen von F. Powi is ${ }^{34}$ ) und H. R. Kruyt ${ }^{35}$ ) bekannt, daB es bei den schwach koagulierenden lonen überhaupt nicht gelingt bis zum Nullwert der Ladung $z u$ gelangen; man bleibt selbst bel großen Konzentrationen - Hunderten von Millimolen - bei Ladungen, die wenig kleiner sind als die, die als Mindestladung zur raschen Koagulation erforderlich ist ${ }^{36}$ ). Bei den stark koagulierenden lonen dagegen wird der Nullwert schon bei kleinen Konzentrationen erreicht oder durchschritten (s. Fig. 3). Man kann demgemäb annehmen, dab bei den schwach koagulierenden lonen die Ladung der Einzelteilchen in den koagulierten Flocken verhältnismäBig hoch bleibt und daB daher die Geschwindigkeit der Vergröberung gering ist; die Flocken bleiben daher langer peptisierbar. Bei den stark koagu-

35) Sven Oden, Der kolloide Schwefel (Nov. Act. Reg. Soc. Scient. Upsala Ser. IV, Vol. lil, Nr. 4, 1913,$157 ;$; H. Freundich a. E. Hase, Zeitschr. f. physik. Chem. 89, 455 (1915); H. Freundllch u. W. Leonhardt, Kolloidchem. Beilh. 7, 183, 203 u. flg. (1915).

A) F. Pow is, loc. cit. S. 166.

d) H. R. Kruyt, Ioc. cit. S. 164.

95) D. h. die Kurve nthert sich bei hoberen Konzentrationen weniger tasch dem Nullpunkt, als es Gleicliung 2 fordern wurde. lierenden lonen sind die Teilchen dagegen so weitgehend entladen, daB auch die weitere Vergröberung überaus schnell verläuft, und man den etwa anfangs vorhandenen Zustand der Peptisierbarkeit gar nicht mehr abfassen kann. Es ist ja bekannt, wie nur bei den schwach knagulierenden lonen dic Flocken zunächst locker und schwammig sind bei den stark koagulierenden lonen sind sie gewöhnlich von vornherein viel dichter und sandiger.

Man wird demgemäB bei den schwach koagulierenden lonen auch nicht hoffen dürfen, in bequem mebbaren Zeiten den Adsorptionsrückgang verfolgen zu können. Da außerdem bei diesen lonen die Veränderlichkeit der Ladung mit der Konzentration sehr klein ist, $\frac{\mathrm{d} \varepsilon}{\mathrm{dc}}$ nahezu null, so wird der Adsorptionsrückgang mit einer fast konstanten geringen Geschwindigkeit vor sich gehen. Gut mebbare, erhebliche Geschwindigkeiten, die sich stark mit der. Konzentration des Elektrolyten ändern, werden nur auftreten, wenn die $\varepsilon$-c-Kurve ziemlich steil den isoelektrischen Punkt durchschneidet (wie in Kurve 3, Fig. 3), also bei stark koagulierenden lonen. In der Tat gelang es bloB bei einigen Parbstoffkationen, die Geschwindigkeit des Adsorptionsrückgangs zu messen, obwoh! aufmerksam nach anderen Fällen gesucht wurde ${ }^{37}$ ). Pür diese Farbstoffkationen ist die starke Adsorbierbarkeit und der steile Verlauf der a-c-Kurve nach elektroosmotischen Versuchen von G. v. Eli ss a f of ${ }^{33}$ ) bekannt.

So lassen sich alle Vergröberungsvorgănge bei kolloiden Teilchen durchaus vom gleichen Gesichtspunkt aus betrachten: 1. der Uebergang äuberst feiner Kolloidteilchen in solche gewöhnlicher GröBe - der oben erwähnte Pall der langsamen Hydrolyse des $\mathrm{FeCl}_{3}-2$ die regelrechte Koagulation kolloider Lösungen, 3. die weitere Vergtöberung koagulierter Flocken beim Adsorptionsruickgang.

Der Umstand, daB im letzten Falle das schliebliche Ergebnis der Umwandlung kleine Kristalle sind $-z$. B. wahrscheinlich beim Adsorptionsrückgang in Schwefelsolen - legt den Gedanken nahe, den auch $R . Z$ sigm o n d ${ }^{39}$ ) auBert, daB die zwischen den Kolloidteilchen wirksamen Krafte dieselben sind, wiè die, welche die Atome im Kristall zusammenhalter. Man

37) H. Freund ich u. E. Hase, Zeitschr. f. physik. Chem. 80, 426 (1915).

3) G. v. Eliss a foft, loc. cit. S. 164.

39) R. Zsigm ondy, Nachr. d. Konigi. Gesellsch. d. Wissensch. Göttingeri 1917, 35. 
möchte dann allgemein die amorph-festen Stoffe als auberst mikrokristallin auffassen, die sog. anisotrop-amorph-festen Stoffe wären feine Kristalle, deren besondere Eigenschaften besonders zu erkiären wăren. Andererseits ist noch immer die Möglichkeit offen, die An ziehung zwischen den Kolloidteilchen als eine Wirkung anzusehen, die der van der Waals. schen Anziehung zwischen den Molekeln der Gase und Flüssigkeiten entspricht. Gewöhnliche wie anisotrop-amorph-feste Stoffe beständen dann noch aus Molekülbauten, und beim Kristallisieren würde z. $T$. das Band der Atome im Molekül zugunsten des Kristallverbandes gelockert, die Atome sprängen teilweise aus den Molekülen in die Punkte des Kristallgitters ein. Eine Eigentümlichkeit des eben genannten Adsorptionsrückganges in Schwefelsolen spricht fast fïr die letztere Auffassung: die koagulierten Schwefeiflöckchen bilden beim Vergröbern erst zähe, ziemlich gut benetzbare, größere Kugeln, die im weiteren Verlauf rasch zu mehr kristallinischen, schlecht benetzbaren Flocken zerfallen, die sich $z$. $T$. an der Oberflache der Flüssigkeit ansammeln ${ }^{40}$ ). -

Die Strenge, mit der man die hier ent wickelte Erklärung des Elektrolyteinflusses bei der Ko.G. und der Geschwindigkeit des Adsorptionsrückganges prüfen konnte, labt noch alles zu wünschen übrig. Es müßte bei einem Sol mit möglichst gleichförmigen Teilchen sowohl die Geschwindigkeit der raschen Koagulation als auch die Abhängigkeit der Geschwindigkeit der nicht raschen Koagulation wie der Teilehenladung von der Elektrolytkonzentration zuverlässig gemessen werden. Dennoch schien es zweckmäBig, diese Betrachtung schon mitzuteilen, weil dieser ElektrolyteinfluB bisher völlig ohne Beispiel dastand, wahrend er jetzt wohl ganz verständlich wird, mag sich die Auffassung im einzelnen auch noch so sehr ändern. Wird ja doch nicht bloB der überaus starke Anstieg der Ko.G. mit wachsender Elektrolytkonzentration erklart, sondern auch das Konstantwerden der Ko.G. bej genügend großen Elektrolytkonzentrationen. -

In der bisherigen Betrachtung ist nur eine Verkleinerung der Ladung von Kolloidteilchen berücksichtigt worden, die durch Erhöhung der Elektrolytkonzentration bewirkt wird. Nun kann die Ladung auch durch sensibilisierende Nichtelektrolyte herabgesetzt werden, so daB bei Elektrolytkonzentrationen, die allein

40) H. Freundll ch u. E.Hase, Zeitschr. f: physik. Chem. 89, 430 (1915). fast keine oder nur eine langsame Koagulation veranlassen würden, bei Gegenwart dieser Nichtelektrolyte eine erhebliche Ko.G. erreicht wird ${ }^{4}{ }^{1}$ ). Diese Erscheinung beruht wahrscheinlich darauf, dab diese Nichtelektrolyte - stark adsorbierbare organische Stoffe wie Urethane, Thymol u. a. - an der Grenzflache der Kolloidteilchen die Dielektrizitătskonstante des Mediums erheblich herabsetzen und dadurch die Ladung verringern. Die oben durchgeführte Theorie macht keine Voraussetzungen darüber, auf welchem Wége die Verkleinerung der Ladung bewirkt wird. Es ist eben, wenn die Ladung durch den sensibilisierenden Stoff verringert wird, eine kleinere lebendige Kraft nötig, damit ein $\mathrm{Zu}$ sammensto $B$ unelastisch wird, die Zahl der wirksamen ZusammenstöBe und somit auch die Ko.G. sind entsprechend grober.

Bei dem Adsorptionsrückgang wird man gleichfalls eine Sensibilisierung erwarten dürfen, nachgewiesen wurde sie bisher noch nicht.

Wenn durch andere Kolloide eine Steigerung oder Verringerung der Ko.G. - Sensibilisierung oder Schutzwirkung - hervorgerufen wird. so werden sich die Verhaltnisse im allgemeinen verwickelter gestalten; aber auch dann wird man unter Umständen die hier erörterten Gesichtspunkte berücksichtigen müssen. -

Auch an dieser Stelle sei darauf bingewiesen, wie wichtig ein Vorgang mit einem derart groben Konzentrationskoeffizienten, wie es die nicht rasche Koagulation in ihrer Elektrolytabhängigkeit ist, wie wichtig ein derartiger Vorgang für die Lebenserscheinungen sein kann. Bei diesen wird die Bedeutung von Koagulationen immer mehr anerkannt, so z. B. bei allen möglichen Hăutchenbildungen, demgemäB bei der Zellteilung, der Befruchtung u. a. m. Nun genügt unter Umständen im Gebiet der nicht raschen Koagulation eine Zunahme der Elektrolytkonzentration von nur 15 Proz., um die Ko.G. zu verdoppeln, eine solche von 50 Proz., um sie zu verdreiBigfachen. Man sieht: Aenderungen des Elektrolytgehaltes einer Zelle, die sich unserer analytischen Genauigkeit fast entziehen, und die sich in anderer Hinsicht kaum geltend machen, können derartige Koagulationsvorgänge sehr beschleunigen; so eben auch die kleinen Stoffmengen, die mit dem Samen ins Ei gelangen, oder die geringen Verschiebungen des Elektrolytgehaltes im Innern des Eies bei der Loeb'schen künstlirhen Befruchtung. Es ist natürlich nicht notwendig, dab es sich allein

1) H. Freundlich u. P. Rona, Biochem. Zeitschr. 81, 87 (1917). 
um eine Aenderung der Elektrolytkonzentration handelt; es kann auch sehr wohl ein Fremdstoff zugeführt werden, der sensibilisierend wirkt, also bei der gleichen Elektrolytkonzentration eine starke Erhöhung der Ko.G. bedingt.

Stark, wenn auch weniger unmittelbar ist der Einflub der Ko.G. bei den Lie segangschen Schichtungen. $\mathrm{Da}$ es sick, hierbei um die Abscheidung schwerlöslicher Stoffe in fester Form handelt, so kommt sowohl die Koagulation wie eine weitere Vergröberung in Frage, bei beiden also auch die entsprechende Einwirkung von Elektrolyten und sensibilisierenden Fremdstoffen. Vielleicht ist der große Konzentrationskoeffizient der Ko.G. geradezu wesentlich für die rhythmische Form derartiger $\mathrm{Ab}$ scheidungen. DaB diese Schichtungen bei den Färbungen der Lebewesen eine entscheidende Rolle spielen, kann als sicher gelten. Nichi weniger wird man also auch auf die Ko.G. und ihre Beeinflussung zurückgteifen müssen, wenn man einst Einzelheiten in der Pigmentierung wird erklären wollen.

\section{Zusammenfassung.}

1. Die Geschwindigkeit der nicht raschen Koagulation hydrophober Sole nimmt auBerordentlich stark mit wachsender Elektrolytkonzentration zu, während bei höheren Elektrolytkonzentrationen im Gebiet der raschen Koagulation die Geschwindigkeit konstant und von der Konzentration und Natur des Elektrolyten una shăngig ist. Die rasche Koagulation hat M. v. S mol u chow ski-molekularkinetisch erklärt, indem er annahm, dab alle ZusammenstöBe zwischen Teilchen unelastisch sind und zur Koagulation führen, bei denen die Teilchen gegenseitig in die Wirkungssphäre ihrer Anziehungskraft gelangen. Um den starken Anstieg der Koagulationsgeschwindigkeit bei der nicht raschen Koagulation zu erklären, wird folgendes angenommen: die in diesem Konzentrationsgebiet vorhandene Ladung der Teilchen bedingt eine Abstoßung; nur die Teilchen gelangen zu einer, die Koagulation bedingenden Berihhrung, deren Geschwindigkeit bzw. I bendige Kraft einen bestimmten kritischen Wert erreicht und übersteigt, der um so größer ist, je größer die
Ladung der Teilchen. Gemäb der Fehlerkurve nimmt die Zahl dieser Teilchen sehr stark $z u_{\text {, }}$ wenn diese kritische Geschwindigkeit mit sinkender Ladung abnimmt, die Elektrolytkonzentration also wächst. Die Formel, die man so für den Zusammenhang zwischen Koagulationsgeschwindigkeit und Elektrolytkonzentration ableiten kann, stimmt mit der Erfahrung überein.

2. Die unter 1 vertretene Anschauung macht es wabrscheinlich, dab der autokatalytische Verlauf für die nicht rasche Koagulation eigentümlich ist und nicht bloB, wie v. Smoluchowski vermutet, von Nebenumständen herrülirt.

3. Auch die Vergrobberung koagulierter Flocken und der damit oft verbundene Adsorptionsrückgang, die bezüglich des autokatalytischen Verlaufes und der Elektrolytabhängigkeit durchaus der Koagulation gleiclien, lassen sich auf Grund der unter 1 entwickelten Auffassung deuten. Die Einzelteilchen in den noch lockeren koagulierten Focken sind nicint völlig entladen und sind ferner $z$. T. durch äuberst feine Flüssigkeitshäutchen getrennt. Die Ladung bedingt wiederum eine Abstoßung, die nur von Teilchen überwunden wird, die in ihren Schwingungen eine gewisse kritische lebendige Kraft erreichen. Blob diese durchbrechen die Flüssigkeitshäutchen und gelangen zur unmittelbaren Berührung und weiteren Verschmelzung, und auf dieser beruht die Vergröberung.

4. Bei Flocken, die mit schwach koagulierenden lonen koaguliert worden sind, ist die Ladung gröber und mit der Elektrolytkonzentration weniger veränderlich als bei Flocken, die mit stark koagulierenden lonen ausgefăllt wurden. Dies hat nach 3 zur Folge, dab sich erstere langsam vergröbern und daher durch Auswaschen unter Umständen peptisieren lassen, während dies bei den letzteren nicht der Fall ist Auch die Tatsache, daB ein gut mebbarer Adsorptionsrückgang nur bei stark adsorbierbaren und stark koagulierenden Farbstoffkationeu benbuchtet wurde, steht mit dieser Theorie im Einklang.

Herrn Dr. E. Oettinger möchte ich für verschiedene Ratschläge in mathematischer Hinsicht auch an dieser Stelle danken. 